\title{
Estilos educativos parentales y emociones como predictores de respuestas obsesivo-compulsivas en población adolescente
}

\section{Parental educational styles and emotions as predictors of obsessive-compulsive responses in adolescent population}

\author{
Ángel Rosa-Alcázar \\ Departamento de Psicología, Universidad Católica de Murcia, España \\ José Luis Parada-Navas \\ Facultad de Educación, Universidad de Murcia, España \\ Pablo J. Olivares-Olivares \\ Cristina Bernal Ruiz \\ Ana I. Rosa-Alcázar \\ Departamento Personalidad, Evaluación y Tratamiento Psicológico, Universidad de Murcia, España
}

Recibido (17 de julio de 2019) Aceptado (10 de octubre de 2019)

\begin{abstract}
Resumen
Las variables familiares y emocionales están relacionadas con el desarrollo y mantenimiento de distintos trastornos psicopatológicos. El objetivo de este estudio fue analizar la relación entre variables emocionales (regulación de emociones, optimismo y tolerancia a la frustración) y familiares (estilos parentales percibidos) con respuestas obsesivo-compulsivas. En cuanto al método, los participantes fueron 473 adolescentes (249 chicos y 224 chicas) con edades comprendidas entre los 12 y 18 años $(M=14.84, D T=1.83)$ que cursaban estudios de Educación Secundaria y Bachillerato. En resultados, la variable optimismo alcanzó el mayor peso explicativo de obsesión-compulsión, seguida de la atención a los síntomas, revelación del padre y tolerancia a la frustración. Las puntuaciones en ansiedad y depresión presentaron un alto porcentaje de varianza explicada. Se concluye que el optimismo, la atención adecuada a los síntomas, la tolerancia a la frustración y las relaciones de confianza entre padres e hijos podrían ser factores protectores en la aparición de pensamientos obsesivo-compulsivos.

Palabras clave: familia, obsesión-compulsión, optimismo, tolerancia a la frustración, regulación emociones.
\end{abstract}

Correspondencia: Ana I. Rosa-Alcázar, Ph.D., Dept. Personalidad, Evaluación y Tratamiento Psicológico, Universidad de Murcia, Facultad de Psicología, Espinardo Campus, Universidad de Murcia, Murcia, 30100, España. Teléfono: +34 868883444; Fax: +34 868 88 4111; Email: airosa@um.es

Agradecimientos: Este artículo es resultado del proyecto concedido por el Ministerio de Economía y Competitividad del Gobierno de España (Proyecto No PSI2016-78185-P) 


\begin{abstract}
Family and emotional variables are related to the development and maintenance of different psychopathological disorders. The aim of this study was to analyze differences between emotional variables (emotion regulation, optimism and tolerance to frustration) and family variables (perceived educational styles) with obsessivecompulsive responses. Method: Participants were 473 adolescents ( 249 boys and 224 girls) aged between 12 and 18 years old $(\mathrm{M}=14.84, \mathrm{SD}=1.83)$ who were studying Secondary Education. Results: The optimism variable reached the highest explanatory weight of obsession-compulsion, followed by attention to symptoms, sharing of feeling with father and tolerance to frustration. The scores in anxiety and depression showed a high percentage of variance explained. Conclusions: Optimism, adequate attention to symptoms, tolerance to frustration and relationships of trust between parents and children could be protective factors of obsessivecompulsive thoughts.
\end{abstract}

Key words: family, obsession-compulsion, optimism, frustration tolerance, emotion regulation

\title{
Introducción
}

El trastorno obsesivo compulsivo (en adelante, TOC) se caracteriza por la presencia de obsesiones y/o compulsiones, que causan ansiedad y malestar significativo en el paciente (American Psychiatric Association, 2013).

Fullana, Mataix-Cold \& Caspi (2009) estimaron que entre el $21 \%$ y $25 \%$ de la población general presentaba signos y síntomas del TOC, estimando la prevalencia del trastorno en niños y adolescentes entre $1 \%$ y $4 \%$ (Douglass, Moffitt, Dar, McGee \& Silva, 1995; Rapoport et al., 2000).

El TOC se ha venido considerando un trastorno unitario, aunque en la clínica se observan grandes diferencias tanto en el contenido de los síntomas como en los patrones de respuesta al tratamiento. Todo esto nos permite plantear la posibilidad de que nos hallemos ante un trastorno con gran heterogeneidad, como apuntan diferentes investigaciones (García-Soriano, Carrió \& Belloch, 2016; Mataix-Cols, do Rosario-Campos \& Leckman, 2005), siendo de gran interés conocer las variables implicadas en la aparición y mantenimiento de la enfermedad.

Diferentes estudios han considerado a la familia como uno de los agentes que puede influir en el origen, mantenimiento o disminución de los síntomas psicopatológicos. La herencia biológica, las alteraciones perinatales, las características temperamentales, la vinculación socioafectiva, la salud familiar, los modelos educativos, las pautas de crianza, etc. ha sido investigadas (Barret, Shortt \& Healy, 2002; Fieldt, Diego \& Sanders, 2001; Kerr \& Stattin, 2000; Santos, Alba, Fernández, Ruibal \& Sudupe, 2013). Castro (2005) informó que un patrón educativo con niveles altos de exigencia y control sin afecto se asociaba a respuestas de ansiedad y depresión en los hijos. La ansiedad infantil se ha relacionado con la sobreprotección, el rechazo e inseguridad de los padres y la disfunción familiar (Breinholst, Esbjørn, Reinholdt-Dunne \& Stallard, 2012). Oliva, Parra, Sánchez \& López (2007) estudiaron las relaciones entre estilos parentales y ajuste del adolescente (problemas internos y externos), destacando la relación existente entre afecto, promoción de autonomía, revelación y humor y buen ajuste emocional; mientras que el control psicológico estaba asociado al desajuste interno y externo. Además, indicaron que los chicos y chicas que percibían más afecto en sus padres y se comunicaban mejor con ellos presentaban mejores puntuaciones en desarrollo psicosocial, bienestar y ajuste emocional, y que existían diferencias significativas a la hora de percibir a los padres y madres, siendo la percepción de las chicas mejor. En esta línea, Rosa-Alcázar, Parada-Navas \& Rosa-Alcázar (2014) informaron que los predictores más relevantes y de mayor peso en la salud psicológica fueron: autoestima, edad, sexo, revelación y afecto del padre y control psicológico de la madre. Además, las chicas percibieron mejor a las madres y a los padres en todas las dimensiones positivas.

Estudios centrados en el entorno familiar del TOC informan de una fuerte relación entre el funcionamiento familiar deficiente y baja eficacia en los resultados de los tratamientos (Garcia et al., 2010; Przeworski et al., 2012; Smorti, 2012). Otras investigaciones destacan la importancia de comprender la salud mental de los padres de niños/adolescentes con TOC (Barrett et al., 2002). 
Los estilos de crianza (clima emocional general en el que se expresan los comportamientos de los padres) y las prácticas de crianza (comportamientos específicos a través de los cuales los padres crían a sus jóvenes) han sido también analizados en pacientes con TOC. Barrett et al. (2002) descubrieron que las madres y los padres de niños con TOC confiaban poco en la capacidad de sus hijos, eran poco afectivos y utilizaban poco el refuerzo educativo. Otros autores han observado altos niveles de control y sobreprotección en familias de adolescentes con TOC (Haciomeroglu \& Karanci, 2014; Timpano, Keough, Mahaffey, Schmidt \& Abramowitz, 2010; Turgeon, O'Connor, Marchand \& Freeston 2002). Timpano et al. (2010) examinaron los estilos de crianza en adultos jóvenes universitarios y hallaron que los síntomas obsesivos compulsivos estaban relacionados con estilos autoritarios caracterizados por bajos niveles de calidez. Estos hallazgos coinciden con otras investigaciones que demuestran una baja percepción de calidez y un mayor rechazo en los padres de pacientes con TOC (Alonso et al., 2001; Lennertz et al., 2010).

Otras investigaciones han girado en torno a la responsabilidad excesiva tanto de los hijos con TOC como de sus madres (Farrell, Hourigan, \& Waters, 2013; Pietrefesa, Schofield, Sochting \& Coles, 2010). El modelo cognitivo del TOC identifica los tipos de experiencias primarias que pueden predisponer a un individuo al desarrollo del trastorno. Salkovskis (1996) afirmaba que las críticas de los padres podían aumentar los síntomas del trastorno obsesivo-compulsivo en los hijos mediante el aprendizaje de una responsabilidad excesiva. Este modelo enfatiza el papel de la crítica y de las prácticas de crianza exigentes como un factor que causa el aumento de creencias y comportamientos obsesivos (Leonard et al., 1993; Tynes, Salins \& Winstead, 1990), siendo el perfeccionismo el modo de evitar la crítica (Rachman, 2002).

Otra variable analizada con el fin de comprender el origen y/o mantenimiento del TOC ha sido el modo de procesar y utilizar la información emocional ya que las personas capaces de percibir, comprender y regular las emociones tienen una mejor adaptación psicológica y social (Salguero, 2011). Estas habilidades han sido conceptualizadas bajo el término Inteligencia Emocional (Mayer \& Salovey, 1997).

Algunas de las dimensiones de la inteligencia emocional son la atención a las emociones, la claridad y la reparación. La atención ha sido definida como la capacidad de tomar conciencia las emociones y valorarlas. La claridad ha sido considerada como la capacidad para identificar, distinguir y describir las experiencias emocionales propias y de los demás. La reparación es definida como la capacidad para regular los estados emocionales de forma correcta (Gohm y Clore, 2000; Salovey, Mayer, Goldman, Turvey \& Palfai, 1995).

Calkins, Berman \& Wilhelm (2013) describieron el mantenimiento de las compulsiones del Trastorno obsesivo-compulsivo desde el modelo de regulación emocional (Rachman, 1997). Cuando el paciente con TOC pretende eliminar los pensamientos/imágenes o impulsos intrusivos que le provocan ansiedad, aumenta la atención prestada a los mismos, impidiéndole su control y llevando a cabo las compulsiones. Park \& Naragon-Gainey (2019) observaron que el grupo TOC presentó problemas en la claridad emocional, en el acceso a estrategias de regulación de emociones y en la aceptación de respuestas emocionales, aun controlando la edad, el nivel educativo, la ansiedad, la depresión y el estrés.

Stern, Nota, Heimberg, Holaway \& Coles (2014) encontraron que los síntomas obsesivos en adolescentes de población no clínica se asociaban con una pobre comprensión emocional y miedo a la ansiedad, ira y depresión, así como a las emociones positivas cuando éstas aparecían de forma inesperada.

Khosravani, Ardestani, Bastan \& Malayeri (2018) hallaron que las dificultades en la regulación de la emociones, especialmente la no aceptación de respuestas emocionales, podían ayudar a mejorar la comprensión del TOC.

El optimismo, sin ser componente de la inteligencia emocional, es un aspecto fundamental para comprender y explicar el comportamiento humano. Se trata de la habilidad para mantener una actitud positiva hacia la vida incluso en situaciones adversas (Carver, 2014). Esta característica personal favorece las interacciones cotidianas con los demás y facilita el manejo de las propias emociones. Estudios centrados en el TOC informaron de la existencia de un sesgo de optimismo irreal en los participantes sanos, mientras que los pacientes con TOC se percibieron más vulnerables a situaciones relacionadas con las obsesiones (Moritz \& Jelinek, 2009; Mousazadeh \& Narimani, 2015; Niemeyer, Moritz \& Pietrowsky, 2013).

Relacionada con el optimismo se encuentra otra habilidad que permite afrontar y manejar las situaciones adversas y estresantes sin sentirse desbordado. Se trata de la Tolerancia a la frustración. Las personas con alta tolerancia se sienten capaces de controlar los impulsos e influir en la situación que provoca estrés, siendo ca- 
paces de actuar ante situaciones difíciles, manteniendo la calma y no dejándose llevar por la desesperanza o la ansiedad (Bernard, Froh, DiGiuseppe, Joyce \& Dryden, 2010). Cougle, Timpano y Goetz (2012) hallaron altos niveles de obsesiones en individuos con baja tolerancia al estrés y dificultad en controlar el impulso. Kashyap et al. (2012) informaron que los sujetos con TOC y con dificultad en el control de impulsos presentaban menor resistencia y control sobre las compulsiones.

Ante esta situación, no hemos hallado estudios centrados en analizar la relación entre los estilos parentales percibidos por los propios adolescentes y diferentes variables emocionales (atención, claridad y reparación emocional; optimismo y tolerancia a la frustración) y las respuestas obsesivo-compulsivas. El objetivo general de nuestro estudio fue analizar dicha relación en población comunitaria adolescente.

Los objetivos específicos fueron: 1) Analizar la relación entre respuestas obsesivo-compulsivas y estilos parentales percibidos y variables emocionales. 2) Examinar la capacidad predictiva de las variables mencionadas en los síntomas obsesivo-compulsivos, observando si es diferente en chicos y chicas. 3) Comprobar si se produce alguna modificación del modelo predictivo cuando se introducen las respuestas ansiosas y depresivas.

\section{Método}

\section{Participantes}

La muestra estuvo formada por un total de 473 adolescentes ( 249 chicos y 224 chicas) con edades comprendidas entre los 12 y los 18 años $(M=14.84, D T=1.83)$ que cursaban estudios de Educación Secundaria y Bachillerato en centros públicos y concertados de las provincias de Murcia y Albacete (España). El 86.3\% de los chicos/as convivían con ambos padres y el $11.3 \%$ sólo con la madre. El $92.6 \%$ de los padres y el $66.8 \%$ de las madres se encontraban trabajando fuera del hogar. Los criterios de inclusión exigidos fueron los siguientes: i) Los participantes no podían presentar trastornos psicopatológicos, como Trastorno de Personalidad, Trastorno del Espectro Esquizofrénico y otros Trastornos Psicóticos, Trastorno bipolar, Anorexia o Bulimia, Trastornos relacionados con dependencia de sustancias y adictivos y Trastornos neurocognitivos. iii) Debían ser mayores de 11 y menores de 19 años. La Tabla 1 recoge una descripción más exhaustiva de las variables sociodemográficas.

Tabla 1. Descripción de variables sociodemográficas y clínicas

\begin{tabular}{|c|c|c|c|}
\hline & $M e d i a \pm D T$ & Frecuencia & Porcentaje \\
\hline Edad (Media $\pm D T)$ & $14.84 \pm 1.83$ & & \\
\hline \multicolumn{4}{|l|}{ Sexo $n(\%)$} \\
\hline Mujer & & 224 & $47.4 \%$ \\
\hline Varón & & 249 & $52.6 \%$ \\
\hline \multicolumn{4}{|l|}{$\mathrm{N}^{\mathrm{o}}$ Hermanos $n(\%)$} \\
\hline Ninguno & & 42 & $8.8 \%$ \\
\hline Uno & & 43 & $9.1 \%$ \\
\hline Dos & & 252 & $53.6 \%$ \\
\hline Tres & & 97 & $20.4 \%$ \\
\hline Cuatro o más & & 39 & $8.1 \%$ \\
\hline \multicolumn{4}{|l|}{ Curso $n(\%)$} \\
\hline $1^{\circ} \mathrm{ESO}$ & & 86 & $18.1 \%$ \\
\hline $2^{\circ} \mathrm{ESO}$ & & 81 & $17.1 \%$ \\
\hline
\end{tabular}




\begin{tabular}{|c|c|c|}
\hline & Frecuencia & Porcentaje \\
\hline $3^{\circ} \mathrm{ESO}$ & 77 & $16.3 \%$ \\
\hline $4^{\circ} \mathrm{ESO}$ & 79 & $16.7 \%$ \\
\hline $1^{\circ}$ Bachillerato & 75 & $15.9 \%$ \\
\hline $2^{\circ}$ Bachillerato & 75 & $15.9 \%$ \\
\hline \multicolumn{3}{|l|}{ Est. Civil Padres $n(\%)$} \\
\hline Casados & 398 & $84.1 \%$ \\
\hline Divorciados/Separados & 62 & $13.1 \%$ \\
\hline Viudos & 7 & $1.5 \%$ \\
\hline Solteros & 6 & $1.3 \%$ \\
\hline \multicolumn{3}{|l|}{ Hogar $n(\%)$} \\
\hline Biparental & 407 & $86 \%$ \\
\hline Monoparental madre & 54 & $11.4 \%$ \\
\hline Monoparental padre & 6 & $1.3 \%$ \\
\hline Otros (abuelos, tíos) & 6 & $1.3 \%$ \\
\hline
\end{tabular}

Nota. $n=$ Número; $D T$ : Desviación típica

\section{Procedimiento}

El procedimiento utilizado fue el siguiente: (1) Envío de una circular a los centros educativos de Educación Secundaria y Bachillerato de las provincias de Murcia y Albacete. (2) Entrevista con los directores de los 8 centros que manifestaron su interés en participar. (3) Selección al azar de un aula por curso (en aquellos centros con doble o triple línea). (4) Envío de una circular a los padres solicitando la autorización para que su hijo/a participara en nuestra investigación. (5) Recibida la autorización, se procedió a la evaluación de los alumnos. Éstos cumplimentaron los cuestionarios de forma anónima en una sesión de una hora de duración en presencia de su profesor y un miembro del equipo de trabajo, experto en evaluación clínica. Los adolescentes no recibieron ningún tipo de refuerzo material por su participación. El estudio cumplió con los criterios éticos informados en la Declaración de Helsinki. También fue aprobado por el Comité de Ética de la Universidad de Murcia (España). Todos los participantes firmaron el consentimiento informado. En la Figura 1 se presenta un diagrama sobre el desarrollo del estudio 


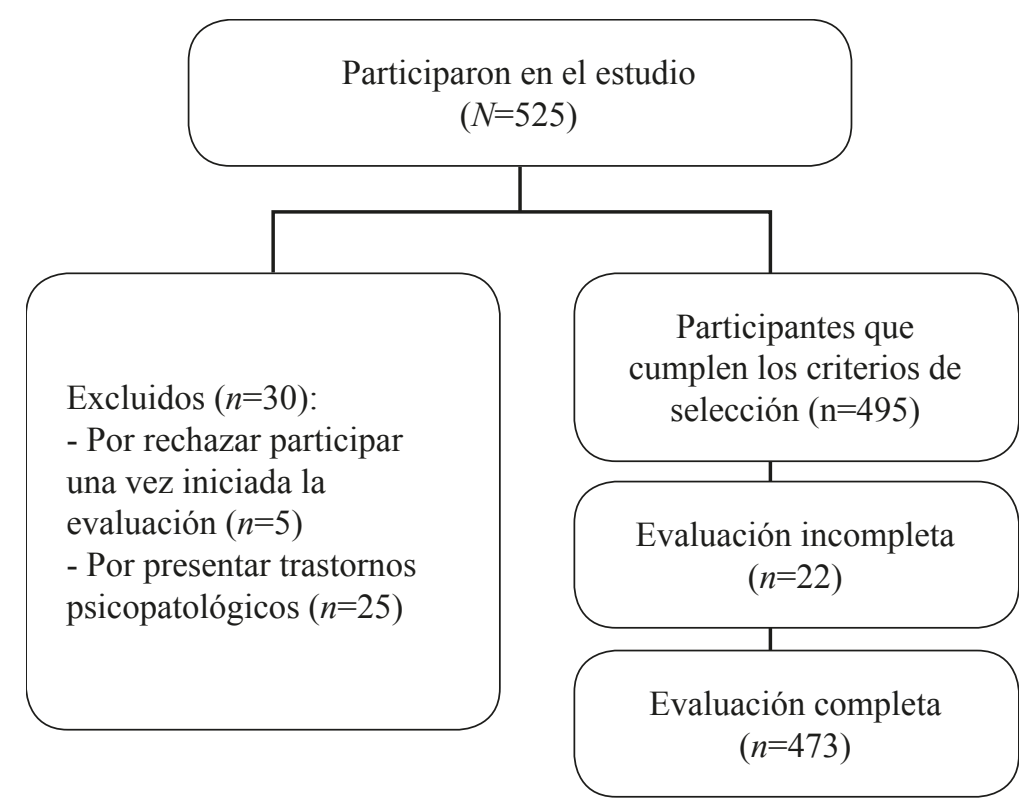

Figura 1. Diagrama CONSORT sobre el desarrollo del estudio

\section{Instrumentos de evaluación}

Los participantes cumplimentaron los siguientes cuestionarios:

» Escala para la evaluación del Estilo parental (Oliva et al., 2007). Consta de 41 ítems en los que el adolescente evalúa de forma independiente 6 dimensiones del estilo materno y paterno. Las dimensiones y su fiabilidad media, calculada mediante la prueba Alpha de Cronbach, fueron las siguientes: Afecto y comunicación $(\alpha=.92)$, Promoción de autonomía $(\alpha=.88)$, Control conductual $(\alpha=.82)$, Control psicológico (dimensión claramente negativa- $\alpha=.86$ ), Revelación $(\alpha=.85)$ y Humor $(\alpha=.88)$. Los índices de fiabilidad en nuestro estudio oscilaron de .80 a .92 .

» Cuestionario de 90 síntomas Revisado (Symptom checklist 90 Revised, SCL-90-R; Derogatis, 1975; adaptación española de González, De las Cuevas, Rodríguez-Abuin \& Rodríguez-Pulido, 2002). Es un cuestionario autoaplicado compuesto por 90 ítems que permite evaluar nueve dimensiones psicopatológicas (somatización, obsesión-compulsión, sensibilidad interpersonal, depresión, ansiedad, hostilidad, ansiedad fóbica, ideación paranoide, psicoticismo), así como tres escalas generales, que son el Índice Global de Gravedad (GSI), el Total de Síntomas Positivos (PST) y el Índice de Distrés de Síntomas Positivos (PSDI). Los índices alfa de fiabilidad de la adaptación española oscilaban entre .77 y .90. Los índices de fiabilidad en nuestro estudio variaron de .79 a .85. Se utilizó la puntuación de la dimensión obsesión-compulsión, depresión y ansiedad.

» Escala para la evaluación del Optimismo (General Mood Subscale, Oliva et al., 2011). Adaptación española de la subescala General Mood Emotional Quotient Inventory (EQ-i, YV, Bar-On \& Parker, 2000). Consta de 8 ítems y evalúa estado de ánimo —optimismo y felicidad— de los niños/as y adolescentes. El índice alfa de Cronbach fue de .91. El índice de fiabilidad en nuestro estudio fue de .89 .

»Escala para la evaluación de la Tolerancia a la Frustración (Stress Management Subscale, Oliva et al., 2011). Adaptación española de Stress Management del Emotional Quotient Inventory (EQ-i, YV, Bar-On \& Parker, 2000). Consta de 8 ítems. La finalidad es evaluar la percepción de los adolescentes sobre su propia capacidad para manejar el estrés-tolerancia a la frustración y la impulsividad. El alfa de Cronbach fue de .77 , siendo .74 en nuestro estudio. 
» Escala para la evaluación de la Expresión, Manejo y Reconocimiento emocional (Trait Meta-Mood Scale, TMMS-48; Salovey et al., 1995), adaptación española de Fernández-Berrocal, Extremera y Ramos (2004). Evalúa la destreza para ser consciente de nuestras propias emociones y la capacidad para regularlas (inteligencia emocional percibida). Los 24 ítems se agrupan en tres dimensiones: Atención emocional (capacidad para sentir y expresar emociones de forma adecuada), Claridad emocional (comprensión de los propios estados emocionales) y Reparación emocional (capacidad para regular los estados emocionales de forma correcta). El índice de fiabilidad de las tres subescalas fue $0.89,0.89$ y 0.85 , respectivamente. El índice de fiabilidad en nuestro estudio fue de $0.86,0.86$ y 0.81 .

\section{Análisis de datos}

El análisis de datos se llevó a cabo usando el paquete estadístico SPSS (V.23.0.) En primer lugar se realizaron ANOVAS o Pruebas $T$ de comparación de medias independientes y pruebas Chi-Cuadrado de Pearson para comprobar si las puntuaciones en obsesiones-compulsiones eran diferentes atendiendo a las variables sociodemográficas. Posteriormente, se analizó la relación entre las respuestas obsesivo-compulsivas y los estilos parentales percibidos y variables emocionales mediante los coeficientes de correlación de Pearson. A continuación, se efectuaron Análisis de regresión por pasos para examinar la capacidad predictiva de diferentes variables sobre la dimensión obsesivo-compulsiva. Todos los participantes fueron incluidos en el análisis.

\section{Resultados}

\section{Respuestas obsesivos-compulsivas y variables sociodemográficas}

El sexo y la edad no alcanzaron diferencias significativas $(p=.965, p=.221$, respectivamente) en la dimensión obsesivo-compulsiva. El resto de variables sociodemográficas ( $n^{\circ}$ de hermanos, situación laboral del padre y de la madre y estado civil de los padres) tampoco hallaron tales diferencias $(p>.05)$

Correlaciones entre respuestas obsesivo-compulsivas, percepción de estilos parentales y variables emocionales

En la Tabla 2 se puede observar que existen relaciones significativas en todas las dimensiones del estilo educativo percibido del padre y de la madre excepto en control conductual del padre $(p=.539)$ y de la madre ( $p$ $=.081$ ), destacando el afecto del padre y de la madre. La variable emocional de mayor relación fue optimismo $(r=-.406, p<.01)$. No presentó relación significativa la variable claridad de las emociones $(p=.213)$. 
Tabla 2. Correlación de Pearson entre percepción de estilos parentales variables emocionales y respuestas obsesivo-compulsivas

\begin{tabular}{lc}
\hline \multicolumn{1}{c}{ Percepción paterna } & Obsesión-compulsión \\
\hline Afecto & $-.226^{* *}$ \\
Promoción & $-.141^{* *}$ \\
Control conductual & -.088 \\
Control psicológico & $.117^{*}$ \\
Revelación & $-.222^{* *}$ \\
Humor & $-.175^{* *}$ \\
$\quad$ Percepción materna & \\
Afecto & $-.231^{* *}$ \\
Promoción & $-.169^{* *}$ \\
Control conductual & -.031 \\
Control psicológico & $.183^{* *}$ \\
Revelación & $-.195^{* *}$ \\
Humor & $-.216^{* *}$ \\
$\quad$ Variables emocionales & \\
Optimismo & $-.406^{* *}$ \\
Tolerancia a la frustración & $-.195^{* *}$ \\
Atención & $.245^{* *}$ \\
Reparación & -.062 \\
\hline & $-.158^{* *}$ \\
\hline & \\
&
\end{tabular}

Variables predictoras de las respuestas obsesivo-compulsivas

En la Tabla 3 se presenta el porcentaje de varianza explicada (coeficientes de determinación ajustados) de las variables predictoras, cuya magnitud fue media-baja (27.1\%), resultando el optimismo el predictor de mayor peso seguida de la atención a los síntomas, revelación del padre y tolerancia a la frustración.

Tabla 3. Resultados del análisis de regresión por pasos de las respuestas obsesivo-compulsivas

\begin{tabular}{llrrl}
\hline & & Beta & \multicolumn{1}{c}{$T$} & $R^{2}$ corregida \\
\hline Obsesión-compulsión & Optimismo & -.340 & $-7.430^{* * *}$ & .271 \\
& Atención & .259 & $5.869^{* * *}$ & \\
& Revelación padre & -.194 & $-4.308^{* * *}$ & \\
& Tolerancia frustración & -.111 & $-2.469^{* * *}$ & \\
\hline
\end{tabular}

Nota. $* * * \mathrm{p}<.001 .{ }^{*} \mathrm{p}<.01 . * \mathrm{p}<.05$

Para comprobar si las variables predictoras de los síntomas obsesivos-compulsivos eran las mismas en ambos sexos, realizamos dos análisis independientes, uno para los chicos y otro para las chicas, llevando a cabo un 
análisis independiente con cada grupo. En la Tabla 4 se puede observar que la variable predictora de mayor peso para ambos sexos fue Optimismo, siendo mayor el porcentaje de varianza explicada en varones que en mujeres.

Tabla 4. Análisis de regresión por pasos de las respuestas obsesivo-compulsivas por sexo

\begin{tabular}{|c|c|c|c|c|}
\hline & & Beta & $\mathrm{T}$ & $\mathrm{R}^{2}$ corregida \\
\hline & Chicos & & & \\
\hline \multirow[t]{10}{*}{ Obsesión-compulsión } & Optimismo & -.428 & $-7.181^{* * *}$ & .341 \\
\hline & Atención & .287 & $4.863^{* * *}$ & \\
\hline & Revelación padre & -.173 & $-2.879^{* * *}$ & \\
\hline & C. Psicológico padre & .143 & $-2.428^{* * *}$ & \\
\hline & Chicas & & & \\
\hline & Optimismo & -.216 & $-3.046^{* * *}$ & .221 \\
\hline & Atención & .282 & $4.115^{* * *}$ & \\
\hline & Revelación padre & -.173 & $-2.531^{* * *}$ & \\
\hline & Claridad & -.168 & $-2.318^{* * *}$ & \\
\hline & Tolerancia frustración & -.136 & $2.052^{* * *}$ & \\
\hline
\end{tabular}

Nota. ${ }^{* *} \mathrm{p}<.001 . * * \mathrm{p}<.01 . * \mathrm{p}<.05$

Relación entre las respuestas ansiosas y depresivas y obsesión-compulsión

En la Tabla 5 se presenta el modelo de regresión por pasos sobre las respuestas obsesivo-compulsivas cuando se introducen las variables familiares y emocionales junto a las puntuaciones en ansiedad y depresión. Se puede observar que la única variable que se mantiene es optimismo ya que ansiedad y depresión alcanzan el mayor peso, explicando estas tres variables un $58 \%$ de varianza.

Tabla 5. Análisis de regresión por pasos de las respuestas obsesivo-compulsiva incluyendo ansiedad y depresión

\begin{tabular}{llccc}
\hline & & Beta & $T$ & $R^{2}$ corregida \\
\hline Obsesión-compulsión & Ansiedad & .497 & $11.174^{* * *}$ & .580 \\
& Depresión & .255 & $5.508^{* * *}$ & \\
& Optimismo & -.152 & $-4.170^{* * *}$ & \\
\hline \multirow{2}{*}{ Nota ${ }^{* * *} \mathrm{p}<.001 . * * \mathrm{p}<.01 . * \mathrm{p}<.05$}
\end{tabular}

Con el fin de comprobar si las respuestas obsesivo-compulsivas se explicaban por los mismos factores en ambos sexo, en la Tabla 6 se presenta el análisis de regresión por pasos para los chicos y para las chicas, siendo mayor el porcentaje de varianza explicada en varones que en mujeres e incluyendo la variable control psicológico del padre. 
Tabla 6. Análisis de regresión por pasos de las respuestas obsesivo-compulsivas incluyendo ansiedad y depresión por sexo

\begin{tabular}{llccc}
\hline & & Beta & \multicolumn{1}{l}{ T } & $R^{2}$ corregida \\
\hline & Chicos & & & \\
\hline Obsesión-compulsión & Ansiedad & .558 & $9.508^{* * *}$ & .660 \\
& Depresión & .210 & $3.581^{* * *}$ & \\
& Optimismo & -.162 & $-3.436^{* * *}$ & \\
& Control psicológico padre & .097 & $2.295^{* * *}$ & \\
\hline & Chicas & & & \\
\hline & Ansiedad & .414 & $6.076^{* * *}$ & .503 \\
& Depresión & .320 & $4.352^{* * *}$ & \\
& Optimismo & -.122 & $-2.130^{* * *}$ & \\
\hline
\end{tabular}

Nota. ${ }^{* * *} \mathrm{p}<.001 . * * \mathrm{p}<.01 . * \mathrm{p}<.05$

\section{Discusión y conclusiones}

El propósito de este estudio fue examinar si los estilos educativos percibidos y diferentes variables emocionales se relacionaban con los síntomas obsesivo-compulsivos en una muestra de adolescentes españoles. Investigaciones previas han examinado la interacción de niños con TOC y sus padres, las pautas de crianza y comunicación, la importancia de los padres en el tratamiento de los niños, la salud mental de los padres, etc. (Barret et al., 2002; Farrell et al., 2013; Lennertz et al., 2010; Pietrefesa et al., 2010; Przeworski et al., 2012; Smorti, 2012; Timpano et al., 2010). No hemos hallado estudios centrados en analizar la relación entre los estilos parentales percibidos por los adolescentes y diferentes variables emocionales (atención, claridad y reparación emocional; optimismo y tolerancia a la frustración) y respuestas obsesivo-compulsivas. Por ello, éste fue el objetivo principal de nuestro estudio. Nuestros resultados indicaron que todas las dimensiones del estilo materno y paterno estuvieron relacionadas, excepto control conductual del padre y de la madre, entendido como el establecimiento de límites y control del comportamiento de los hijos fuera de casa. El afecto del padre y de la madre estuvieron relacionados de forma negativa, de decir, a mayor sensación de apoyo y afecto, disponibilidad y fluidez en la comunicación con los padres, menores respuestas obsesivas. Estos resultados apoyan investigaciones previas realizadas con población clínica, donde la calidez emocional, la aceptación en niños con TOC era baja (Alonso et al., 2001; Lennertz et al., 2010; Timpano et al., 2010). Otra de las dimensiones de mayor relación fue revelación o confianza para comentar a sus padres/madres lo que les sucede, no sintiendo miedo al castigo o rechazo siendo así más autónomos, yendo en la dirección de lo apuntado por otros autores (Leonard et al., 1993; Tynes, Salins \& Winstead, 1990). En general, las dimensiones de los estilos parentales positivas están relacionadas con menos respuestas obsesivas, siendo el control psicológico por parte del padre, entendido como conductas de manipulación y culpa, una dimensión relacionada con más obsesiones-compulsiones.

Las variables emocionales se relacionaron con obsesión-compulsión, excepto claridad de las emociones de la Inteligencia emocional. Tomar conciencia de las emociones, valorarlas y regularlas permite disminuir los niveles de malestar, aprender a tolerar o posponer las decisiones. Mantener una actitud positiva ante las situaciones, ayudará a gestionar mejor el mundo emocional, controlando así las respuestas obsesivas. No obstante, tal y como indicó Calkins et al. (2013), un exceso de atención a las emociones conllevaría un aumento de ansiedad y miedo a los pensamientos impidiéndole su control y realizando, por ello, más compulsiones.

El segundo de nuestros objetivos pretendió examinar la capacidad predictiva de la percepción de los estilos parentales y las variables emocionales, observando que las que mejor explicaban los síntomas obsesivo-compulsivos fueron la aptitud positiva ante los sucesos que ocurren cada día, la atención media a los síntomas que provocan ansiedad, la confianza con el padre y la capacidad de tolerar y resistir situaciones o sucesos estresantes y demorar los impulsos, apoyando este resultado lo obtenido en el estudio de Cougle et al. (2012). Nuestros 
resultados informaron que el mayor numero de variables implicadas eran emocionales.

Teniendo en cuenta estudios previos en los que se informa de la heterogeneidad del TOC teniendo en cuenta la variable sexo (Olatunji, Sawchuk, Arrindell \& Lohr, 2005), la diferente percepción encontrada en los estilos parentales percibidos por adolescentes (Rosa et al., 2014) y las diferencias en emociones y cogniciones (Moser, Moran, Kneip, Schroder \& Larson, 2016), nos llevó a plantearnos si los predictores en obsesión-compulsión serían los mismos en los chicos que en las chicas. Se observó un mayor porcentaje de varianza explicada con menos predictores en los chicos (optimismo, atención, revelación del padre y control psicológico del padre) que en las chicas (optimismo, atención, revelación del padre, claridad y tolerancia). Ello nos lleva a concluir que la claridad de las emociones o capacidad para identificar, distinguir y describir las experiencias emocionales junto a la tolerancia a la frustración son variables relevantes en las mujeres, mientras que en los varones lo es el control o manipulación del padre. Por tanto, el sexo podría ser una variable a tener en cuenta no sólo en el TOC clínico sino también en participantes con respuestas obsesivo-compulsivas.

La alta comorbilidad de la depresión y ansiedad con el TOC (Fullana et al., 2009) junto a importancia de estas respuestas en la regulación de las emociones (Park \& Naragon-Gainey, 2019; Stern et al., 2014) nos llevó a plantearnos el tercero de nuestros objetivos. Éste consistió en comprobar si se producía alguna modificación en el modelo predictivo de los síntomas obsesivo-compusivos cuando se introducían las respuestas ansiosas y depresivas de los adolescentes. Observamos que la variable ansiedad, seguida de depresión y optimismo explicaba un $58 \%$ de los síntomas obsesivo-compulsivos. Esto no nos permite indicar que la ansiedad y depresión provocan obsesiones y compulsiones sino que, tal y como se observa en la clínica, los adolescentes con obsesiones-compulsiones, tras resistirse a las mismas comienzan a generar mayores respuestas depresivas y ansiosas. Resultados parecidos obtuvimos en el modelo analizado para chicos y chicas, incluyendo en el caso de los chicos la variable control psicológico o manipulación del padre. Este resultado podría ir en la dirección de lo informado por Salkovskis (1996) ya que las conductas manipuladoras de los padres suelen provocar culpa en los hijos llevándoles a intentar comportarse como los padres desean como modo de evitar la crítica.

Los resultados de este estudio nos llevan a concluir que las variables emocionales, en concreto el bajo optimismo, la excesiva atención a las emociones y la baja tolerancia a la frustración pueden ser factores de riesgo que potencien la aparición de respuestas obsesivo-compulsivas. Como implicación clínica sería importante que el adolescente aprendiera a gestionar sus emociones y a percibirlas de forma adecuada. La terapia cognitivoconductual podría ayudar a comprender cuándo se desencadena la emoción y la relación entre el sentimiento, pensamientos y conducta. Aprender estas habilidades, teniendo como modelo a los padres, podría ser un factor protector de salud para los niños y adolescentes. Por ello, la confianza o revelación con el padre, evitando el chantaje emocional podría mejorar las interacciones familiares siendo éstas más positivas y flexibles, impidiendo así el reforzamiento de conductas inadecuadas (Peris \& Piacentini, 2013).

Estos resultados hemos de interpretarlos teniendo en cuenta las limitaciones principales de este estudio. Se trata de un diseño de carácter transversal y correlacional lo que nos impide hablar de relación causal. Otra limitación ha sido que sólo hemos contado con autoinformes de los adolescentes. Por otra parte, no hemos utilizado medidas concretas que evalúen el TOC, ni la depresión o ansiedad, sino que nos hemos centrado en una medida que mide sólo síntomas, por tanto, no pueden generalizarse los resultados ni a población clínica ni se está informando de trastornos sino de síntomas.

Estudios futuros podrían mejorar lo informado ampliando el tamaño muestral e incluyendo medidas de comportamiento, fisiológicas o informes de terceros. Además, se podrían realizar estudios longitudinales sobre el mantenimiento de las respuestas obsesivo-compulsivas durante varios periodos temporales o comparar las variables contemplas en el estudio en diferentes trastornos, con el fin de comprender si se tratan de factores de riesgo específicos del TOC o, por el contrario, de procesos transdiagnósticos.

\section{Referencias}

Alonso, M.P., Menchon, J.M., \& Pifarre, J. (2001). Estudio de la percepción del estilo educativo en el trastorno obsesivo-compulsivo. Psiquiatria Biologica, 8, 94-99.

American Psychiatric Association (2013). Diagnostic and statistical manual of mental disorders (DSM-5). Washington, D.C.: American

Psychiatric Association. 
Barrett, P., Shortt, A., \& Healy, L. (2002). Do parent and child behaviours differentiate families whose children have obsessive-compulsive disorder from other clinic and non-clinic families? Journal of Child Psychology and Psychiatry, 43, 597-607.

Bar-On, R., \& Parker, J. D. A. (2000). The Bar-On EQ-i: YV: Technical manual. Toronto: Multi-Health Systems.

Bernard, M.E., Froh, J. J., DiGiuseppe, R., Joyce, M.R., \& Dryden, W. (2010). Albert Ellis: Unsung hero of positive psychology. The Journal of Positive Psychology, 5, 302-310.

Breinholst, S., Esbjørn, B. H., Reinholdt-Dunne, M. L., \& Stallard, P. (2012). CBT for the treatment of child anxiety disorders: A review of why parental involvement has not enhanced outcomes. Journal of Anxiety Disorders, 26, 416-424. doi:10.1016/j.janxdis.2011.12.014

Calkins, A. W., Berman, N. C., \& Wilhelm, S. (2013). Recent advances in research on cognition and emotion in OCD: a review. Current Psychiatry Reports, 15, 357. doi: 10.1007/s11920-013-0357-4

Carver, C. S. (2014). Optimism. En A. Michalos (ed.), Encyclopedia of quality of life and well-being research (pp. 4500-4503). Springer: NY

Castro, J. (2005) Disciplina y estilo educativo familiar. En L. Ezpeleta (ED). Factores de riesgo en psicopatología del desarrollo (pp. 319-336). Barcelona: Masson.

Cougle, J. R., Timpano, K. R., \& Goetz, A.R. (2012). Exploring the unique and interactive roles of distress tolerance and negative urgency in obsessions. Personality and Individual Differences, 52, 515-520. doi:10.1016/j.paid.2011.11.017

Derogatis, L. R. (2002). SCL-90-R. Cuestionario de 90 síntomas revisado. Madrid: TEA (original work published in 1983)

Douglass, H.M., Moffitt, T.E., Dar, R., McGee, R. O. B., \& Silva, P. (1995). Obsessive-compulsive disorder in a birth cohort of 18 -yearolds: prevalence and predictors. Journal of the American Academy of Child and Adolescent Psychiatry, 34, 1424-1431.

Farrell, L. J., Hourigan, D., \& Waters, A. M. (2013). Do mothers enhance responsibility in children with obsessive compulsive disorder? A preliminary study of mother-child interactions during a problem solving discussion. Journal of Obsessive-Compulsive and Related Disorders, 2, 78-84. doi:10.1016/j.jocrd.2012.12.001

Fernández-Berrocal, P., Extremera, N., \& Ramos, N. (2004). Validity and reliability of the Spanish modified version of the Trait Meta-Mood Scale. Psychological Reports, 94, 751-755.

Field, T., Diego, M., \& Sanders, C. (2001). Adolescent depression and risk factors. Adolescence, 36, 491-499.

Fullana, M., Mataix-cold, A., \& Caspi, H. (2009). Obsession and compulsion in the community: prevalence, interference, help-seeking, developmental stability and co-occuring psychiatric conditions. American Journal of Psychiatry, 166, 329-336. doi:10.1176/appi. ajp.2008.08071006

Garcia, A. M., Sapyta, J. J., Moore, P. S., Freeman, J. B., Franklin, M.E., March, J. S., \& Foa, E. B. (2010). Predictors and moderators of treatment outcome in the Pediatric Obsessive Compulsive Treatment Study (POTS I). Journal of the American Academy of Child and Adolescent Psychiatry, 49, 1024-1033. doi:10.1016/j.jaac.2010.06.013

García-Soriano, G., Carrió, C., \& Belloch, A. (2016). Psicopatología de las compulsiones de lavado en el trastorno obsesivo-compulsivo: no todos los pacientes lavan por los mismos motivos. Revista de Psicopatología y Psicología Clínica, 21, 219-230. doi:10.5944/rppc. vol.21.num.3.2016.15901

Gohm, C. L., \& Clore, G. L. (2000). Individual differences in emotional experience: Mapping available scales to processes. Personality and Social Psychology Bulletin, 26, 679-697. doi:10.1177/0146167200268004

González, J.L., De las Cuevas, C., Rodríguez-Abuín, M., \& Rodríguez-Pulido, F. (2002). SCL-90-R, Symptom Cheklist 90 Revised. Spanish adaptation. Madrid: TEA.

Haciomeroglu, B., \& Karanci, A. N. (2014). Perceived parental rearing behaviours, responsibility attitudes and life events as predictors of obsessive compulsive symptomatology: Test of a cognitive model. Behavioural and Cognitive Psychotherapy, 42, 641-652. doi:10.1017/s1352465813000581

Kashyap, H., Fontenelle, L.F., Miguel, E. C., Ferrão, Y A., Torres, A. R., Shavitt, R. G., ... \& Yücel, M. (2012). Impulsive compulsivity in obsessive-compulsive disorder: A phenotypic marker of patients with poor clinical outcome. Journal of Psychiatric Research, 46, 1146-1152. doi:10.1016/j.jpsychires.2012.04.022

Kerr, M., \& Stattin, H. (2000). What parents know, how they know it, and several forms of adolescent adjustment: further support for a reinterpretation of monitoring. Developmental psychology, 36, 366. doi:10.1037//0012-1649.36.3.366

Khosravani, V., Ardestani, S. M. S., Bastan, F. S., \& Malayeri, S. (2018). Difficulties in emotion regulation and symptom dimensions in patients with obsessive-compulsive disorder. Current psychology, 1-11. doi:10.1007/s12144-018-9859-x

Lennertz, L., Grabe, H. J., Ruhrmann, S., Rampacher, F., Vogeley, A., Schulze-Rauschenbach, S., ... \& Pukrop, R. (2010). Perceived parental rearing in subjects with obsessive-compulsive disorder and their siblings. Acta Psychiatrica Scandinavica, 121, $280-288$. doi:10.1111/j.1600-0447.2009.01469.x

Leonard, H.L., Swedo, S. E., Lenane, M. C., Rettew, D. C., Hamburger, S., \& Bartko, J. J. (1993). A two to seven year follow up study of 54 obsessive compulsive children and adolescents. Archives of General Psychiatry, 50, 429-439. doi:10.1001/archpsyc.1993.01820180023003 
Mataix-Cols, D., do Rosario-Campos, M.C., \& Leckman, J.F. (2005). A multidimensional model of obsessivecompulsive disorder. American Journal of Psychiatry, 162, 228-238. doi:10.1176/appi.ajp.162.2.228

Mayer, J.D., \& Salovey, P. (1997). What is emotional intelligence? En P. Salovey y D.J. Sluyter (Eds.), Emotional development and emotional intelligence: Implications for educators (pp. 3-31). New York: Basic Books

Moritz, S., \& Jelinek, L. (2009). Inversion of the "unrealistic optimism" bias contributes to overestimation of threat in obsessive-compulsive disorder. Behavioural and Cognitive Psychotherapy, 37, 179-193. doi:10.1017/s1352465808005043

Moser, J. S., Moran, T. P., Kneip, C., Schroder, H. S., \& Larson, M. J. (2016). Sex moderates the association between symptoms of anxiety, but not obsessive compulsive disorder, and error-monitoring brain activity: A meta-analytic review. Psychophysiology, 53, 21-29. doi:10.1111/psyp.12509

Mousazadeh, T., \& Narimani, M. (2015). The Role of Styles of Thinking and Attributional Styles and Optimism and Pessimism in the Prediction of Obsessive-Compulsive Disorder. Scientific Journal of Ilam University of Medical Sciences, 23, 125-133.

Niemeyer, H., Moritz, S., \& Pietrowsky, R. (2013). Responsibility, metacognition and unrealistic pessimism in obsessive-compulsive disorder. Journal of Obsessive-Compulsive and Related Disorders, 2, 119-129. doi:10.1016/j.jocrd.2013.01.001

Olatunji, B. O., Sawchuk, C.N., Arrindell, W. A., \& Lohr, J.M. (2005). Disgust sensitivity as a mediator of the sex differences in contamination fears. Personality and Individual Differences, 38, 713-722.

Oliva, A., Antolín, L., Pertegal, M. A., Ríos, M., Parra, A., Hernando, A., \& Reina, M. C. (2011). Instrumentos para la evaluación de la salud mental y el desarrollo positivo adolescente y los activos que lo promueven. Junta de Andalucía: Consejería de Salud.

Oliva, A., Parra, A., Sánchez, I., \& López, F. (2007). Estilos educativos materno y paterno: evaluación y relación con el ajuste del adolescente. Anales de Psicología, 25, 49-56.

Park, J., \& Naragon-Gainey, K. (2019). Is more emotional clarity always better? An examination of curvilinear and moderated associations between emotional clarity and internalising symptoms. Cognition and Emotion, 1-15. doi: 10.1080/02699931.2019.1621803

Peris, T. S., \& Piacentini, J. (2013). Optimizing treatment for complex cases of childhood obsessive compulsive disorder: a preliminary trial. Journal of Clinical Child y Adolescent Psychology, 42, 1-8. doi:10.1080/15374416.2012.673162

Pietrefesa, A. S., Schofield, C. A., Sochting, I., \& Coles, M.E. (2010). Obsessive beliefs in youth with OCD and their mothers. Journal of Cognitive Psychotherapy, 24, 187-197. doi:10.1891/ 0889-8391.24.3.187

Przeworski, A., Zoellner, L. A., Franklin, M.E., Garcia, A., Freeman, J., March, J. S., \& Foa, E. B. (2012). Maternal and child expressed emotion as predictors of treatment response in pediatric obsessive-compulsive disorder. Child Psychiatry and Human Development, 43, 337-353. doi:10.1007/s10578-011-0268-8

Rachman, S. (1997). A cognitive theory of obsessions: Elaborations. Behaviour Research and Therapy, 35, 793-802. doi:10.1016/s00057967(97)00040-5

Rachman, S. (2002). A cognitive theory of compulsive checking. Behaviour Research and Therapy, 40, 625-639. https://doi.org/10.1016/ s0005-7967(01)00028-6

Rapoport, J. L., Inoff-Germain, G., Weissman, M. M., Greenwald, S., Narrow, W.E., Jensen, P. S., ... \& Canino, G. (2000). Childhood obsessive-compulsive disorder in the NIMH MECA Study: Parent versus child identification of cases. Journal of Anxiety Disorders, 14, 535-548.https://doi.org/10.1016/s0887-6185(00)00048-7

Rosa-Alcázar, A. I., Parada-Navas, J.L., \& Rosa-Alcázar, Á. (2014). Síntomas psicopatológicos en adolescentes españoles: relación con los estilos parentales percibidos y la autoestima. Anales de Psicología/Annals of Psychology, 30, 133-142. doi:10.6018/analesps.30.1.165371

Sahmelikoglu O., Tabo, A., Aydin, E., Tuna, O., Maner, A. F., Yildirim, E. A., \& Çarpar, E. (2016). Relationship between impulsivity and obsession types in obsessive-compulsive disorder. International Journal of Psychiatry in Clinical Practice, 20, 218-223.

Salguero, M. J.C. (2011). Importancia de la inteligencia emocional como contribución al desarrollo integral de los niños/as de educación infantil. Pedagogía Magna, 11, 178-188.

Salovey, P., Mayer, J. D., Goldman, S.L., Turvey, C., \& Palfai, T.P. (1995). Emotional attention, clarity and repair: Exploring emotional intelligence using the Trait Meta-Mood Scale. En J. W. Pennebaker (Ed.), Emotion, disclosure and health (pp. 125-154). Washington, DC: American Psychological Association. doi:10.1037/10182-006

Salkovskis, P. M. (1996). Cognitive-behavioral approaches to the understanding of obsessional problems. Current Controversies in the Anxiety Disorders, 103-133.

Santos, M.G., Alba, J. V., Fernández, E. R., Ruibal, F. J. O., \& Sudupe, J. A. (2013). Trastorno obsesivo-compulsivo (TOC) genética y ambiente. A propósito de una experiencia clínica. Revista gallega de psiquiatría y neurociencias, 12, 156-158.

Smorti, M. (2012). The impact of family on obsessive compulsive disorder in children and adolescents: development, maintenance, and family psychological treatment. International Journal of Advances in Psychology, 3, 86-94. 
Stern, M. R., Nota, J. A., Heimberg, R. G., Holaway, R. M., \& Coles, M.E. (2014). An initial examination of emotion regulation and obsessive compulsive symptoms. Journal of Obsessive-compulsive and Related Disorders, 3, 109-114. doi: 10.1016/j.jocrd.2014.02.005

Timpano, K. R., Keough, M.E., Mahaffey, B., Schmidt, N. B., \& Abramowitz, J. (2010). Parenting and obsessive compulsive symptoms: Implications of authoritarian parenting. Journal of Cognitive Psychotherapy, 24, 151-164.doi:10.1891/0889-8391.24.3.151

Tynes, L. L., Salins, C., \& Winstead, D. K. (1990). Obsessive compulsive patients: familial frustration and criticism. The Journal of the Louisiana State Medical Society: official organ of the Louisiana State Medical Society, 142, 24-6.

Turgeon, L., O’Connor, K.P., Marchand, A., \& Freeston, M.H. (2002). Recollections of parent-child relationships in patients with obsessive-compulsive disorder and panic disorder with agoraphobia. Acta Psychiatrica Scandinavica, 105, 310-316. doi:10.1034/ j.1600-0447.2002.1188.x 